\title{
Inhibition of dendritic cell maturation and activation is mediated by
} STAT3

\author{
K Bauer*1, S Binder ${ }^{1}$, C Klein ${ }^{2}$, JC Simon² and F Horn ${ }^{1}$
}

Address: ${ }^{1}$ University of Leipzig, Department of Molecular Immunology, Leipzig, Germany and ${ }^{2}$ University of Leipzig, Department of Dermatology, Venerology and Allergology, Leipzig, Germany

* Corresponding author

from 12th Joint Meeting of the Signal Transduction Society (STS). Signal Transduction: Receptors, Mediators and Genes

Weimar, Germany. 29-31 October 2008

Published: 26 February 2009

Cell Communication and Signaling 2009, 7(SuppI I):A68 doi:10.1186/1478-8IIX-7-SI-A68

This abstract is available from: http://www.biosignaling.com/content/7/SI/A68

(c) 2009 Bauer et al; licensee BioMed Central Ltd.

Dendritic cells (DC) are the most potent antigen presenting cells, because they can internalize, process and present antigens in order to activate T-cells. Accordingly, DC has the ability to activate tumour-specific T-cell response. However, this does not occur in most types of cancer, in opposite DC even help maintaining tumour tolerance. Instead of being eliminated by the immune response, tumours progress and metastasize. This failure of the immune system is caused by an abnormal DC differentiation and activation mediated through several tumour derived factors like IL- 6 and IL-10. It is well known, that IL-6 and IL-10 signalling is mainly supported by signal transducer and activator of transcription 3 (STAT3). The mechanisms how STAT3 affects the maturation and activation of DC and which STAT3 target genes are involved in this process is still unknown.

To understand the modulation of dendritic cell function by IL-10, gene expression profiling was performed by using Affymetrix technology in human monocyte-derived DC treated with IL-10, alone or in combination with LPS. The regulation of selected genes was validated by real-time PCR. IL-10 regulated in DC the expression of a limited number of genes, including IL-7, CD200, DGKA and signal transduction elements. We have compared the cell phenotype and functional properties of monocyte/macrophage derived dendritic cells which where preincubated with IL-10 followed by activation through LPS. This combined treatment modulated a number of genes comparable to LPS alone, but differ in their amplitude of regulation. As expected, IL-10 suppressed the expression of several LPS-inducible proinflammatory molecules.

Our results deliver insight into, how STAT3 interferes with maturation and activation of DC and generates immunotolerance towards tumours. 